\title{
Multi-Wavelength Surveys for Galaxies Hidden by the Milky Way
}

\author{
Renée C. Kraan-Korteweg \\ Departamento de Astronomía, Universidad de Guanajuato, Guanajuato \\ GTO 36000, Mexico
}

\begin{abstract}
The systematic mapping of obscured and optically invisible galaxies behind the Milky Way through complementary surveys are important in arriving at the whole-sky distribution of complete galaxy samples and therewith for our understanding of the dynamics in the local Universe. In this paper, a status report is given of the various deep optical, near infrared (NIR), and systematic blind $\mathrm{H}$ I-surveys in the Zone of Avoidance, including a discussion on the limitations and selection effects inherent to the different multi-wavelength surveys and first results.
\end{abstract}

\section{Introduction}

Due to the foreground extinction of the Milky Way, galaxies become increasingly fainter, smaller and are of lower surface brightness as they approach the Galactic Equator. Although most of them are not intrinsically of low surface brightness, "whole-sky" mapping of galaxies is required (a) in explaining the origin of the peculiar velocity of the Local Group (LG) and the dipole in the Cosmic Microwave Background, (b) for our understanding of velocity flow fields such as the Great Attractor in the Zone of Avoidance (ZOA) with a predicted mass excess of a few times $10^{16} \mathrm{M}_{\odot}$ at $(\ell, b, v) \sim\left(320^{\circ}, 0^{\circ}, 4500 \mathrm{~km} \mathrm{~s}^{-1}\right.$, Kolatt et al. 1995), and (c) other suspected connections of nearby superclusters and voids behind the Milky Way.

This not only concerns large-scale structures. Nearby massive galaxies behind the obscuration layer of the Milky Way could significantly change our understanding of the internal dynamics and mass derivations of the LG.

Dedicated searches for galaxies in about $25 \%$ of the optically obscured extragalactic sky so far revealed a number of important features such as:

- the nearby bright spiral galaxy Dwingeloo 1, a neighbor to the LG (KraanKorteweg et al. 1994)

- the Puppis cluster at $(\ell, b, v) \sim\left(245^{\circ}, 0^{\circ}, 1500 \mathrm{kms}^{-1}\right)$ which may contribute at least $30 \mathrm{~km} \mathrm{~s}^{-1}$ to the motion of the LG perpendicular to the Supergalactic Plane (Lahav et al. 1993)

- the massive Coma-like cluster A3627 at $(\ell, b, v) \sim\left(325^{\circ},-7^{\circ}, 4800 \mathrm{~km} \mathrm{~s}^{-1}\right)$ which seems to constitute the previously unrecognized but predicted density peak at the bottom of the potential well of the Great Attractor (Kraan-Korteweg et al. 1996) 
- the $3 \mathrm{C} 129$ cluster at $(\ell, b, v) \sim\left(160^{\circ}, 0^{\circ}, 5500 \mathrm{~km} \mathrm{~s}^{-1}\right)$ connecting PerseusPisces and A569 across the Galactic Plane (Chamaraux et al. 1990, Pantoja et al. 1997)

- and the Ophiuchus (super-)cluster at $(\ell, b, v) \sim\left(0^{\circ}, 8^{\circ}, 8500 \mathrm{~km} \mathrm{~s}^{-1}\right)$ behind the Galactic Center (Wakamatsu et al. 1994).

In the following, I will review the current status of deep optical searches behind the Milky Way, as well as the possibilities and results given with the recent near infrared surveys and blind H I-surveys.

\section{Optical Surveys}

Systematic optical galaxy catalogs are generally limited to the largest galaxies (typically with diameters $\mathrm{D} z 1^{\prime}$, e.g., Lauberts 1982). These catalogs become, however, increasingly incomplete as the dust thickens, creating a "Zone of Avoidance" in the distribution of galaxies of roughly $25 \%$ of the sky. Systematic deeper searches for partially obscured galaxies - down to fainter magnitudes and smaller dimensions compared to existing catalogs - were performed with the aim to reduce this $\mathrm{ZOA}$. These surveys are not biased with respect to any particular morphological type.

The various survey regions are displayed in Fig. 1 (cf. Woudt 1998, for an extensive overview). Further details and results on the uncovered galaxy distributions can be found in A: Aquila and Sagittarius (Roman et al. 1996), B: Sagittarius/Galactic (Roman \& Saito 1997), C: Ophiuchus Supercluster (Wakamatsu et al. 1994), D: Galactic Center extension (Kraan-Korteweg, in progress), E: Crux and GA Region (Woudt \& Kraan-Korteweg 1999, in prep.), F: Hydra/Antlia Supercluster (Kraan-Korteweg 1999, in prep.), G: Hydra to Puppis Region (Salem \& Kraan-Korteweg, in progress) H \& I : Puppis (Saito et al. 1990, 1991), I: Perseus-Pisces Supercluster (Pantoja 1997), J : northern crossing GP/SGP (Hau et al. 1996), K: northern ZOA (Seeberger et al. 1994).

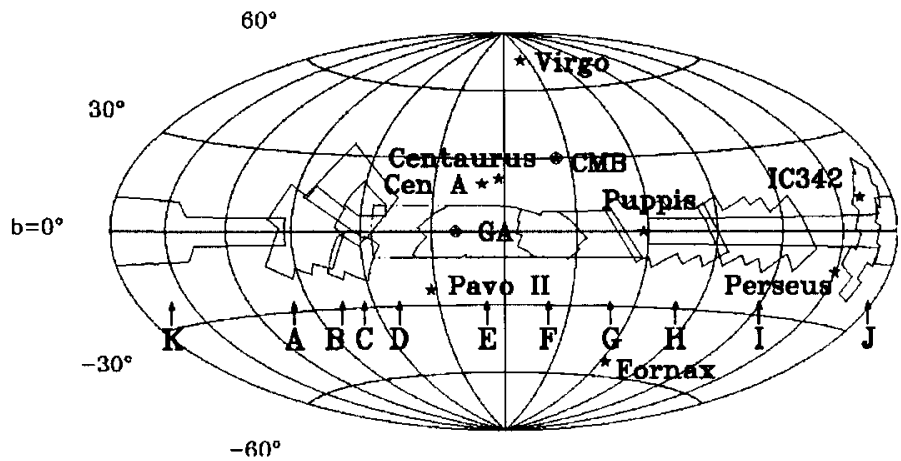

Figure 1. An overview of the different optical galaxy surveys in the ZOA centered on $\ell=300^{\circ}$. The labels identifying the search areas are explained in the text. Galaxy cluster positions (stars) and the CMB apex as well as the core of the GA are marked. 
Meanwhile, as Fig. 1 illustrates, nearly the whole ZOA has been surveyed systematically. With the mapping of over 50000 previously unknown galaxies a considerable reduction of the ZOA was achieved. Analysing the galaxy density as a function of the galaxy size, magnitude and/or morphology in combination with the foreground extinction has led to the identification of various important large-scale structures. However, redshift follow-ups of well-defined samples are important in tracing the large-scale structures in detail. Such follow-up surveys have already revealed a number of dynamically important structures in the Zone of Avoidance ( $c f$. Introduction).

Although the various optical surveys are based on different plate material and the criteria for inclusion in the respective surveys were not all identical, all surveys reveal the same dependence on extinction: for extinctions in the blue of $A_{B} \gtrsim 4-5^{m}$, the ZOA remains fully opaque (cf. top panel of Fig. 3), leaving a strip of about $\pm 5^{\circ}$ devoid of galaxies.

\section{NIR-Surveys}

The extinction effects decrease with increasing wavelengths. In the NIR passbands $I_{c}, J$ and $K_{s}$, the extinction compared to the blue is $A_{I_{c}}=45 \%, A_{J}=$ $21 \%$, and $A_{K_{s}}=9 \%$. Moreover, NIR surveys are sensitive to early-type galaxies - tracers of massive groups and clusters missed in IRAS and H I surveys - and have little confusion with Galactic objects. Here, the recent near infrared surveys, 2MASS (Skrutskie et al. 1997) and DENIS (Epchtein 1997) might provide new insight at low Galactic latitudes.

In unobscured regions, the density of galaxies per square degree for the completion limit of $B_{J} \leq 19^{m} 0$ is 110 (Gardner et al. 1996). However, the number counts in the blue decrease rapidly with increasing obscuration as $N\left(A_{\mathrm{B}}\right) \simeq$ $110 \times \operatorname{dex}\left(0.6\left[-A_{\mathrm{B}}\right]\right) \mathrm{deg}^{-2}$. In the NIR passbands $I_{c}, J$ and $K_{s}$ of the DENIS survey, the counts for the respective completeness limits of $I_{\lim }=16^{m} 0$, $J_{\lim }=14^{m} 0, K_{\lim }=12^{m} 2$ are considerably lower $(30,11$, and 2 , Mamon et al. 1997) but - as illustrated in Fig. 2 - the decrease in number counts as a function of "optical extinction" is considerably slower. The new cooling system for the focal instrument of DENIS installed in 1997 led to an increase in the $K_{s}$ band counts of a factor of two for the completeness limit fainter by $\sim 0^{m} 5$.

Fig. 2 shows that the NIR becomes notably more efficient where the Milky Way becomes opaque in the optical $\left(A_{B} \geq 4-5^{\mathrm{m}}, c f\right.$. previous section). At an extinction of $A_{B} \simeq 3-4^{\mathrm{m}}, J$ becomes superior to $I_{c}$, while at $A_{B} \simeq 10^{\mathrm{m}}, K_{s}$ becomes superior to $J$. The cooled camera system will make the $K_{s}$ passband competitive with $J$ starting at $A_{B} \simeq 7^{\mathrm{m}}$. These are very rough predictions and do not take into account any dependence on morphological type, surface brightness, orientation and crowding, which may lower the counts of actually detectable galaxies counts.

\subsection{First Results from DENIS}

To compare these predictions with real data, Schröder et al. (1997) and KraanKorteweg et al. (1998a) examined the efficiency of uncovering galaxies at high extinctions with DENIS images. The results are promising. 


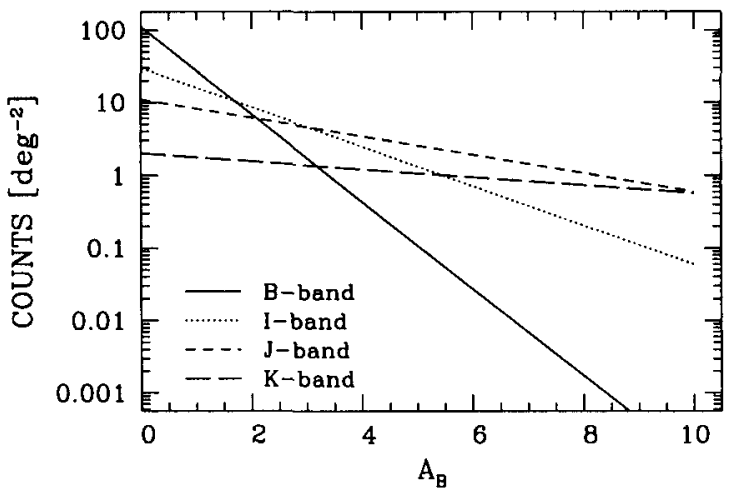

Figure 2. Predicted galaxy counts in $B, I_{c}, J$ and $K_{s}$ as a function of absorption in $B$, for highly complete and reliable DENIS galaxy samples and a $B_{J} \leq 19^{\mathrm{m}}$ optical sample.

They found that down to intermediate latitudes and extinction $\left(|b| \gtrsim 5^{\circ}\right.$, $A_{B} \lesssim 4-5^{\mathrm{m}}$ ), optical surveys remain superior for identifying galaxies. However, the NIR luminosities and colors together with extinction data from the NIR colors will prove invaluable in analysing the optical survey data and their distribution in redshift space, and in the final merging of these data with existing sky surveys. Despite the high extinction and the star crowding at these latitudes, $I_{c}, J$ and $K_{s}$ photometry from the survey data can be successfully performed at these low latitudes and led, for instance, to the preliminary $I_{\mathfrak{c}}^{\circ}, J^{\circ}$ and $K_{s}^{\circ}$ galaxy luminosity functions in A3627.

At low latitudes and high extinction $\left(|b|<5^{\circ}\right.$ and $\left.A_{B} \geq 4-5^{\mathrm{m}}\right)$, the search for 'invisible' obscured galaxies on existing DENIS-images implicate that NIRsurveys can trace galaxies down to about $|b| \simeq 1.5$. The $J$ band was found to be optimal for identifying galaxies up to $A_{B} \simeq 7^{\mathrm{m}}$, although this might change in favour of $K_{s}$ with the new cooling system. NIR surveys can hence further reduce the width of the $\mathrm{ZOA}$. Furthermore, this is the only tool that permits the mapping of early-type galaxies - tracers of density peaks - at high extinction.

The analysis of DENIS images behind the ZOA is being pursued in a more systematic way. Whether this will be performed by visual examination or whether galaxies can be successfully extracted using classical algorithms or artificial neural networks or a combination of both requires further exploration.

\section{Blind H I surveys}

In the regions of the highest obscuration and infrared confusion, the Galaxy is fully transparent to the $21-\mathrm{cm}$ line radiation of neutral hydrogen. $\mathrm{H}$ l-rich galaxies can readily be found at lowest latitudes through detection of their redshifted 21-cm emission. Only low-velocity extragalactic sources (blue- and redshifted) within the strong Galactic $\mathrm{H}$ I emission will be missed, and - because of baseline ripple - galaxies close to radio continuum sources. Until recently, radio receivers were not sensitive and efficient enough to attempt large systematic surveys of 
the ZOA. In a pilot survey with the late $300-\mathrm{ft}$ telescope of Green Bank, Kerr \& Henning (1987) surveyed $1.5 \%$ of the ZOA and detected 16 new spiral galaxies.

\subsection{The Northern Zone of Avoidance}

Using the Dwingeloo $25 \mathrm{~m}$ radio telescope, the whole northern Galactic ZOA $\left(|b| \leq 5^{\circ} 25\right)$ is being surveyed in the $21 \mathrm{~cm}$ line for galaxies out to $4000 \mathrm{~km} \mathrm{~s}^{-1}$ (see also Rivers et al., these proceedings). A shallow search ( $\mathrm{rms}=175 \mathrm{mJy}$ ) has been completed yielding five objects (Henning et al. 1998). This fast search for nearby massive galaxies uncovered no major unknown Andromeda-like galaxy. The most exciting discovery is the barred spiral galaxy Dwingeloo 1 (KraanKorteweg et al. 1994), a new neighbour of the Local Group with one third of the Galaxy's mass.

The deeper survey (rms=40 mJy) is $60 \%$ complete. 36 galaxies were detected of which 23 were previously unknown, the most surprising being the detection of a number of dwarfs at very low redshifts. They lie close to the Sdm galaxy NGC 6946 at v=48 $\mathrm{km} \mathrm{s}^{-1}$, suggesting a previously unrecognized nearby group or cloud of galaxies.

\subsection{The Southern Zone of Avoidance}

In March 1997, a systematic blind H I survey began in the the southern Milky Way $\left(|b| \leq 5^{\circ}\right.$ ) with the multibeam (MB) receiver (13 beams in the focal plane array) at the $64 \mathrm{~m}$ Parkes telescope. The survey covers the velocity range $-1200 \lesssim v \lesssim 12700 \mathrm{~km} \mathrm{~s}^{-1}$ and will have a sensitivity of $\mathrm{rms}=6 \mathrm{mJy}$ after Hanning smoothing.

So far, a shallow survey based on 2 out of the foreseen 25 driftscan passages has been analysed (cf. Henning et al. these proceedings, and KraanKorteweg et al. 1998b). 107 galaxies were catalogued with peak H I-flux densities of $\chi^{80 ~ J y ~ k m ~ s}{ }^{-1}$ ( $\mathrm{rms}=15 \mathrm{mJy}$ after Hanning smoothing). Though galaxies up to $6500 \mathrm{~km} \mathrm{~s}^{-1}$ were identified, most of the galaxies $(80 \%)$ are quite local $\left(\mathrm{v}<3500 \mathrm{~km} \mathrm{~s}^{-1}\right.$ ) due to the (yet) low sensitivity.

Most detections are due to normal two-horned spiral galaxies. However, ATCA follow-up observations of three very extended $\left(20^{\prime}\right.$ to $\left.\chi 1^{\circ}\right)$, nearby $(v<$ $1500 \mathrm{~km} \mathrm{~s}^{-1}$ ) sources revealed them to be interesting galaxies/complexes, with unprecedented low H I column densities (cf. Staveley-Smith et al. 1998).

As in the northern $\mathrm{H}$ I-survey, no Andromeda or other $\mathrm{HI}$-rich Circinus galaxy has been found lurking undetected behind the extinction layer of the southern Milky Way. Both H I-surveys have, however, clearly proven the power of tracing spiral and $\mathrm{H}$ I-rich dwarf galaxies through the deepest extinction layer of the Milky Way (cf. Fig. 3 and 4, as well as Fig. 2 in Rivers et al., these proceedings).

\section{Conclusions}

Considerable progress has been made in mapping the galaxy distribution with various multi-wavelength approaches. The continuing surveys will lead to a much more complete picture of the galaxy distribution in the "former" ZOA. 


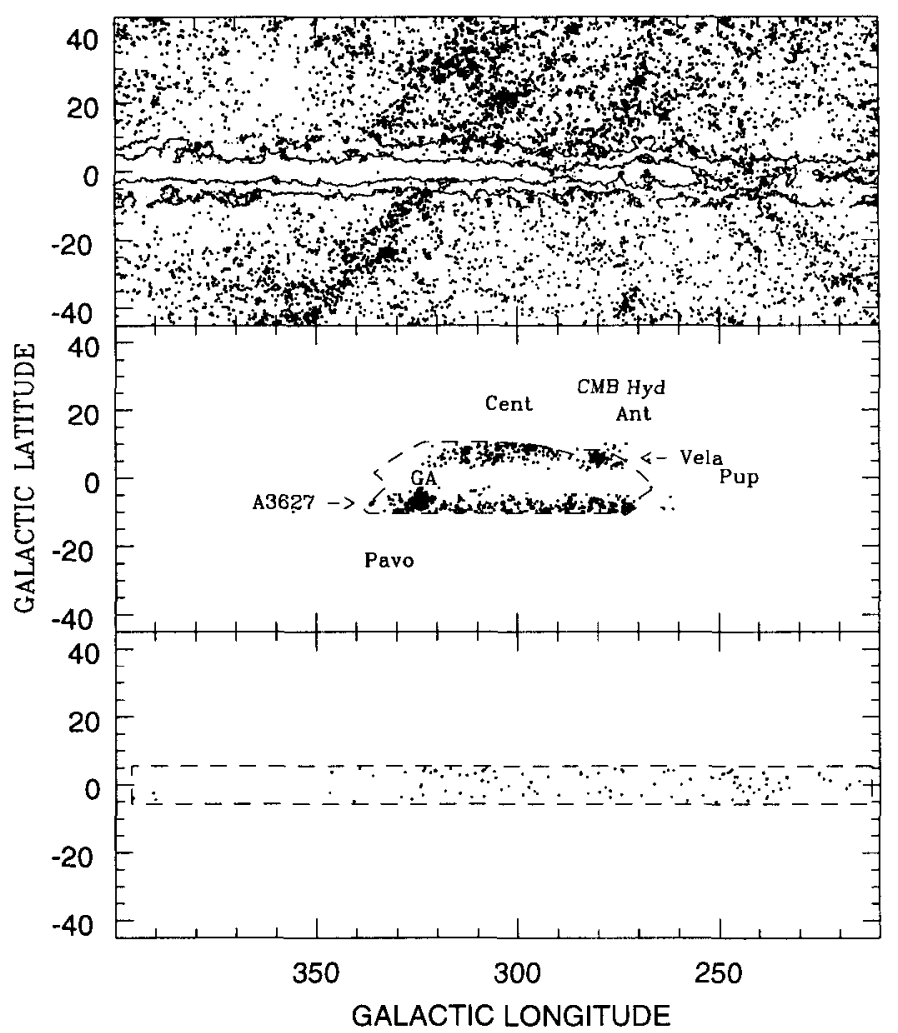

Figure 3. Galaxies with $\mathrm{v}<10000 \mathrm{~km} \mathrm{~s}^{-1}$. Top panel: literature values (LEDA), superimposed are extinction levels $A_{B} \sim 1^{m} 5$ and $5^{m}$; middle panel: follow-up redshifts (ESO, SAAO and Parkes) from deep optical ZOA survey with locations of clusters and dynamically important structures; bottom panel: redshifts from shallow $\mathrm{MB}-\mathrm{ZOA}$ in $\mathrm{HI}$ with the Parkes radio telescope.

How complementary the various multi wavelength approaches are is illustrated in Fig. 3. The top panel shows the distribution of all known galaxies with velocities $\mathrm{v} \leq 10000 \mathrm{~km} \mathrm{~s}^{-1}$ centered on the southern Milky Way. Although this constitutes an uncontrolled sample, it traces the main structures in the nearby Universe in a representative way. Note the near full lack of galaxy data for extinction levels $A_{B} \sim 1$. $^{m} 5$ (outer contour).

The middle panel results from the follow-up observations of the optical galaxy search by Kraan-Korteweg and collaborators. Various new overdensities could be unveiled at intermediate extinction levels and low latitudes $\left(1^{m} 5 \lesssim\right.$ $\left.A_{\mathrm{B}} \lesssim 5^{m}, 5^{\circ} \lesssim|b| \lesssim 10^{\circ}\right)$, but the innermost part of our Galaxy remains obscured $\left(A_{B} \gtrsim 4-5^{m},|b| \lesssim 5^{\circ}\right)$. Here, the blind $\mathrm{HI}$ data finally provide the missing link for large-scale structure studies as indicated with the results from the detections in the shallow survey of the Parkes MB survey (lower panel). 


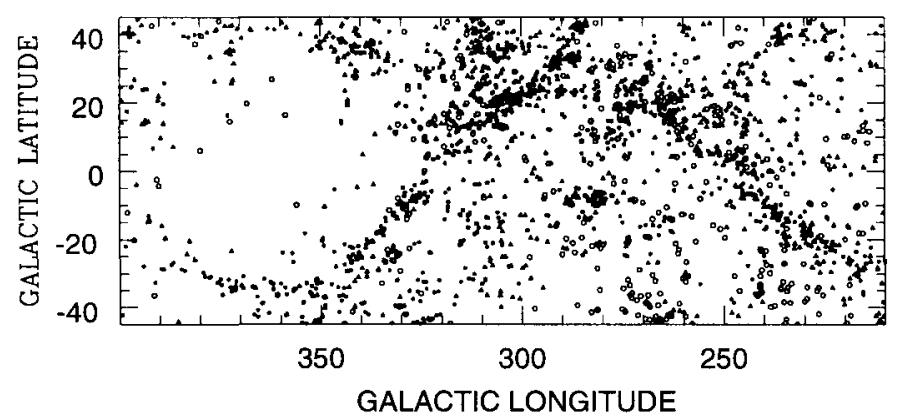

Figure 4. Redshift slices from data in Fig. 3 for the velocity range $500<\mathrm{v}<3500 \mathrm{~km} \mathrm{~s}^{-1}$. The open circles mark the nearest $(500-1500)$, the triangles the medium $(1500-2500)$ and the filled dots the most distant (2500-3500) slice.

In Fig. 4, the data from the various surveys displayed in Fig. 3 are combined for the redshift range $500<\mathrm{v}<3500 \mathrm{~km} \mathrm{~s}^{-1}$. The upper velocity limit reflects the depth achieved with the sensitivity of the shallow MB H I-survey. For the first time, structures are visible all the way across the ZOA. Note the continuity of the thin filamentary sine-wave-like structure that dominates the whole southern sky, and the prominence of the Local Void. With the full sensitivity of the MBsurvey, we will be able to fill in the large-scale structures out to $10000 \mathrm{~km} \mathrm{~s}^{-1}$.

The possibilities given for ZOA-research based on the currently ongoing NIR are very promising - and complementary in the sense that they finally allow the uncovering of early-type galaxies to low Galactic latitudes $(|b| \geq 1-1.5)$. This is not the only addition NIR surveys provide. Schröder et al. (1997) and KraanKorteweg et al. (1998a) have shown that a fair fraction of heavily obscured spiral galaxies detected in blind $\mathrm{H}$ I surveys can be reidentified on DENIS images ( $c f$, Fig. 5 in Kraan-Korteweg et al. 1998a). The combination of H I data with NIR data allow the study of the peculiar velocity field via the NIR Tully-Fisher relation "in the ZOA" compared to earlier interpolations of data adjacent to the ZOA (Schröder et al, in progress).

A difficult task still awaiting us in the future is the merging of ZOA data with catalogs outside the ZOA. This will have to be done with care to obtain 'unbiased' whole-sky surveys.

Acknowledgments. The collaborations with my colleagues in the various multi-wavelength surveys - P.A. Woudt with the optical surveys, A. Schröder and G.A. Mamon in the exploration of the DENIS survey, W.B. Burton, P.A. Henning, O. Lahav and A. Rivers in the northern ZOA H I-survey (DOGS) and the HIPASS ZOA team members R.D. Ekers, A.J. Green, R.F. Haynes, P.A. Henning, S. Juraszek, M. J. Kesteven, B. Koribalski, R.M. Price, E. Sadler and L. Staveley-Smith in the southern ZOA survey - are greatly appreciated. 


\section{References}

Chamaraux P., Cayatte V., Balkowski C., Fontanelli P. 1990, A\&A 229, 340

Epchtein N. 1997, in The Impact of Large Scale Near-Infrared Surveys p. 15, eds. F. Garzon et al., Kluwer, Dordrecht

Gardner J.P., Sharples R.M., Carrasco B.E., Frenk C.S. 1996, MNRAS 282, L1

Hau G.K.T., Ferguson H.C., Lahav O. et al. 1996, MNRAS 277, 125

Henning P.A., Kraan-Korteweg R.C., Rivers A.J. et al. 1998, AJ 115, 584

Kerr F.J., Henning P.A. 1987, ApJ 320, L99

Kolatt T., Dekel A., Lahav O. 1995, MNRAS 275, 797

Kraan-Korteweg R.C., Loan A.J., Burton W.B. et al. 1994, Nature 372, 77

Kraan-Korteweg R.C., Woudt P.A., Cayatte V. et al. 1996, Nature 379, 519

Kraan-Korteweg R.C., Schröder A., Mamon G., Ruphy S. 1998a, in The Impact of Near-Infrared Surveys on Galactic and Extragalactic Astronomy, p.205, ed. N. Epchtein, Kluwer, Dordrecht

Kraan-Korteweg R.C., Koribalski B., Juraszek S. 1998b, in Looking Deep in the Southern Sky, eds. R. Morganti, W. Couch, Springer, in press

Lahav O., Yamada T., Scharf C.A., Kraan-Korteweg, R.C. 1993, MNRAS 262, 711

Lauberts, A. 1982, The ESO/Uppsala Survey of the ESO (B) Atlas, ESO, Garching

Mamon, G.A., Tricottet, M., Bonin, W., Banchet, V., 1997, in Extragalactic Astronomy in the Infrared, p. 369, eds. G. A. Mamon et al., Frontières, Paris

Pantoja C.A., Altschuler D.R., Giovanardi C., Giovanelli R. 1997, AJ 113, 905

Roman A.T., Nakanishi K., Tomita A., Saito M. 1996, PASJ 48, 679

Roman A.T., Nakanishi K., Saito M. 1998, PASJ 50, 37

Saito M., Ohtani A., Asomuna A. et al. 1990, PASJ 42, 603

Saito M., Ohtani A., Baba A. et al. 1991, PASJ 43, 449

Schröder A., Kraan-Korteweg R.C., Mamon G.A. Ruphy S. 1997, in Extragalactic Astronomy in the Infrared, p. 381, eds. G. A. Mamon et al., Frontières, Paris

Seeberger R., Saurer W., Weinberger R. et al. 1994, in Unveiling Large-Scale Structures Behind the Milky Way, p. 81, eds. C. Balkowski, R.C. KraanKorteweg, ASP Conf. Ser. 67

Skrutskie, M.F., et al. 1997, in The Impact of Large Scale Near-Infrared Surveys, p. 25, eds. F. Garzon et al., Kluwer, Dordrecht

Staveley-Smith L., Juraszek S., Koribalski B.S. et al. 1998, AJ, in press

Tully R.B., Fisher J.R. 1977, A\&A 54, 66

Wakamatsu K., Hasegawa T., Karoji H. et al. 1994, in Unveiling Large-Scale Structures Behind the Milky Way, p. 131, eds. C. Balkowski, R.C. KraanKorteweg, ASP Conf. Ser. 67

Woudt P.A. 1998, Ph.D. thesis, Univ. of Cape Town. 